\title{
POSITIVE SOLUTIONS OF NONLINEAR SECOND ORDER ORDINARY DIFFERENTIAL EQUATIONS
}

\author{
Z. ZHAO
}

(Communicated by Hal L. Smith)

\begin{abstract}
We prove an existence theorem for positive solutions of nonlinear second order ordinary differential equations by using the perturbation method and the Schauder fixed point theorem.
\end{abstract}

We consider the following problem on the nonlinear second order ordinary differential equation in $R^{+}=(0, \infty)$ :

$$
\left\{\begin{array}{l}
\text { (1a) } u^{\prime \prime}+f(x, u)=0, \\
\text { (1b) } u>0 \text { in } R^{+}, \\
\text {(1c) } \lim _{x \rightarrow 0^{+}} u(x)=0,
\end{array}\right.
$$

where $f(x, y)$ is a Borel measurable function in $R^{+} \times R^{+}$and dominated by a positive function $F$ :

$$
|f(x, y)| \leq F(x, y), \quad(x, y) \in R^{+} \times R^{+} .
$$

The main goal of this paper is to prove an existence theorem for problem (1) under certain conditions on $F$.

For each $x \in R^{+}, y \rightarrow F(x, y)$ is a convex and continuously differentiable function in $[0, \infty)$ such that

$$
F(x, 0)=F_{y}(x, 0)=0
$$

and

$$
\int_{0}^{\infty} x F_{y}(x, x) d x<\infty .
$$

Theorem 1. Let $f(x, y)$ be a Borel measurable function of $(x, y)$ in $R^{+} \times R^{+}$ and a continuous function of $y$ in $R^{+}$for each fixed $x \in R^{+}$. If there exists a dominated function $F$ in $R^{+} \times[0, \infty)$ satisfying (3) and (4), then problem (1) has infinitely many solutions. More precisely, there exists a number $b>0$ such that for each $c \in(0, b]$, (1) has a solution $u$ satisfying

$$
\lim _{x \rightarrow \infty} \frac{u(x)}{x}=c \text {. }
$$

Received by the editors September 14, 1992.

1991 Mathematics Subject Classification. Primary 34A34, 47H10; Secondary 60J45. 
Remark. The domain $R^{+}$can be replaced by any half-line $(a, \infty)$ or $(-\infty, a)$ with trivial changes.

A special but important case of the above general setting is the semilinear problem:

$$
\left\{\begin{array}{l}
u^{\prime \prime}+K(x) u^{p}=0 \text { in } R^{+}, p>1, \\
u>0 \text { in } R^{+} \\
\lim _{x \rightarrow 0^{+}} u(x)=0 .
\end{array}\right.
$$

Remark. There exist great interest and intensive studies for positive solutions of high dimensional elliptic semilinear problems of the type of problem (6); see, e.g., [1].

For any Borel measurable function $K$ in $R^{+}$, if we take $f(x, y)=K(x) y^{p}$ and $F(x, y)=|K(x)| y^{p}$, then $f$ and $F$ satisfy (2) and (3) obviously. Condition (4) on $F$ is equivalent to

$$
\int_{0}^{\infty} x^{p}|K(x)| d x<\infty .
$$

Am immediate consequence of Theorem 1 is

Theorem 2. For any Borel measurable function $K$ in $R^{+}$satisfying (7), the conclusion of Theorem 1 holds for problem (6).

In [3], the author proved an existence theorem for the nonlinear elliptic equations with dimension $d \geq 3$. Theorem 1 can be regarded as its one dimensional version, but the functiom $f(\cdot, \cdot)$ here has a more general form, which means higher nonlinearity, and the conditions of $f(\cdot, \cdot)$ are simpler. We shall present a purely analytical proof for Theorem 1 instead of a probabilistic potential theory approach as in [3]. At the end of this paper, we will show a probabilistic explanation of some key steps in the proof, for the benefit of readers interested in probabilistic methods. In fact, both methods, analytic and probabilistic ones, inspire and enlighten each other.

Proof of Theorem 1. By convexity and differentiability of $F(x, \cdot)$ and $(3)$, we conclude that both $F(x, \cdot)$ and $F_{y}(x, \cdot)$ are nonnegative and nondecreasing in $R^{+}$for each $x \in R^{+}$. Then by (3), (4) and the dominated convergence theorem,

$$
\lim _{\beta \downarrow 0} \int_{0}^{\infty} x F_{y}(x, \beta x) d x=0 .
$$

Hence we can fix a number $\beta>0$ such that

$$
\int_{0}^{\infty} x F_{y}(x, \beta x) d x<\frac{1}{3} \text {. }
$$

Let

$$
\begin{aligned}
& C[0, \infty]=\left\{v: v \text { is a continuous function in } R^{+}\right. \text {such that } \\
& \left.\qquad \lim _{x \rightarrow 0^{+}} v(x) \text { and } \lim _{x \rightarrow \infty} v(x) \text { exist }\right\} .
\end{aligned}
$$

It is easy to see that $C[0, \infty]$ is a Banach space with the norm

$$
\|v\|=\sup _{x \in R^{+}}|v(x)| \text {. }
$$


Let $b=\frac{2}{3} \beta$. For each fixed $c \in(0, b]$, let

$$
\Lambda=\left\{v \in C[0, \infty]: c / 2 \leq v(x) \leq 3 c / 2 \text { for all } x \in R^{+}\right\}
$$

For each $v \in \Lambda$, we construct a linear operator in $C[0, \infty]$ :

$$
A_{v} \psi(x)=\frac{1}{x} \int_{0}^{\infty}(x \wedge y) \frac{f(y, v(y) y)}{v(y)} \psi(y) d y, \quad \psi \in C[0, \infty],
$$

where $x \wedge y=\min (x, y)$. Since $\|v\| \leq 3 b / 2=\beta$, we have by (3) and the mean value theorem:

$$
\left|\frac{f(y, v(y) y)}{v(y)}\right| \leq y F_{y}(y, \beta y) .
$$

Hence it follows from the dominated convergence theorem and (8) that $A_{v} \psi \in$ $C[0, \infty]$ and

$$
\left\|A_{v}\right\| \leq \int_{0}^{\infty} y F_{y}(y, \beta y) d y<\frac{1}{3}
$$

Let

$$
T v(x)=c \sum_{n=0}^{\infty}\left(A_{v}^{n} 1\right)(x), \quad x \in R^{+} .
$$

Then (11) implies that $T v \in C[0, \infty]$ and for each $x \in R^{+}$we have

$$
T v(x) \leq c \sum_{n=0}^{\infty}\left\|A_{v}\right\|^{n} \leq \frac{3}{2} c
$$

and

$$
T v(x) \geq c\left(1-\sum_{n=1}^{\infty}\left\|A_{v}\right\|^{n}\right) \geq \frac{c}{2} .
$$

Therefore $T v \in \Lambda$; that is, $T$ is a mapping from $\Lambda$ to itself. By (12), $T$ satisfies

$$
T v=c+A_{v}[T v]
$$

i.e., $\forall x \in R^{+}$

$$
T v(x)=c+\frac{1}{x} \int_{0}^{\infty}(x \wedge y) \frac{f(y, v(y) y)}{v(y)}(T v)(y) d y
$$

Then for any $x$ and $z$ in $R^{+}$, we have by (10) and (13):

$$
|T v(x)-T v(z)| \leq \frac{3 c}{2} \int_{0}^{\infty}\left|\frac{x \wedge y}{x}-\frac{z \wedge y}{z}\right| y F_{y}(y, \beta y) d y .
$$

By the dominated convergence theorem, we have

$$
T v(\infty) \stackrel{\text { def }}{=} \lim _{x \rightarrow \infty} T v(x)=c
$$

and

$$
T v(0) \stackrel{\text { def }}{=} \lim _{x \rightarrow 0^{+}} T v(x)=c+\int_{0}^{\infty} y \frac{f(y, v(y) y)}{v(y)}(T v)(y) d y
$$


Hence

$$
|T v(x)-T v(\infty)| \leq \frac{2 c}{3 x} \int_{0}^{\infty}(x \wedge y) y F_{y}(y, \beta y) d y
$$

and

$$
|T v(x)-T v(0)| \leq \frac{2 c}{3} \int_{0}^{\infty}\left|\frac{x \wedge y}{x}-y\right| y F_{y}(y, \beta y) d y
$$

It follows from (13), (16), (19), and (20) that the family of functions $\{T v: v \in$ $\Lambda$ \} is uniformly bounded and equicontinuous in $C[0, \infty]$; hence it is relatively compact in $C[0, \infty]$.

We next prove that $T$ is a continuous mapping in $\Lambda$. Let $\left\{v_{k}\right\}$ in $\Lambda$ such that $\left\|v_{k}-v\right\| \rightarrow 0$ as $k \rightarrow \infty$. Since $f$ is continuous with the second variable, it follows from (9), (10), and the dominated convergence theorem that $\forall x \in R^{+}$, $n \geq 1, \psi \in C[0, \infty]$

$$
A_{v_{k}}^{n} \psi(x) \rightarrow A_{v}^{n} \psi(x) \quad \text { as } k \rightarrow \infty
$$

Then by using (11) and (12) we have

$$
T_{v_{k}}(x) \rightarrow T v(x) \text { as } k \rightarrow \infty
$$

that is, the sequence of functions $\left\{T_{v_{k}}\right\}$ converges to $T v$ pointwisely. Since we have proved that $\left\{T v_{k}\right\}$ is relatively compact in $C[0, \infty]$, pointwise convergence implies uniform convergence, namely, $\left\|T v_{k}-T v\right\| \rightarrow 0$ as $k \rightarrow \infty$, we thus proved the continuity of $T$. Therefore $T$ is a compact operator in $\Lambda$ (see [2, Definition 2.9]). It is obvious from the definition (9) of $\Lambda$ that $\Lambda$ is a nonempty, bounded, closed, and convex set in $C[0, \infty]$. Hence by the Schauder fixed point theorem (see [2, Theorem 2.A]) there exists a function $v_{0} \in \Lambda$ such that

$$
T v_{0}=v_{0}
$$

This together with (15) implies that

$$
v_{0}(x)=c+\frac{1}{x} \int_{0}^{\infty}(x \wedge y) f\left(y, v_{0}(y) y\right) d y
$$

Put

$$
u(x)=x v_{0}(x)
$$

We then have by (22) that

$$
\begin{gathered}
u(x)=c x+\int_{0}^{\infty}(x \wedge y) f(y, u(y)) d y \\
=c x+\int_{0}^{x} y f(y, u(y)) d y+x \int_{x}^{\infty} f(y, u(y)) d y \\
u^{\prime}(x)=c+\int_{x}^{\infty} f(y, u(y)) d y
\end{gathered}
$$

and

$$
u^{\prime \prime}(x)=-f(x, u(x))
$$

Thus $u$ satisfies equation (1a). Conditions (1b) and (1c) follow from (23) obviously, and (5) follows from (22) and (23). 
Remark. Any solution $u>0$ of (1a) can be regarded as a solution of the following Schrödinger equation:

$$
u^{\prime \prime}+q u=0 \quad \text { with } q=f(x, u) / u
$$

in $(0, \infty)$. This suggests that one could use the Feynman-Kac formula to change the differential equation (1a) to another equation for $u$ (not to a representation of solution since $q$ also depends on $u$ !). The zero boundary condition (1c) suggests using the conditional Brownian motion: $x$-process of Doob's type (note that $h(x) \equiv x$ is a positive harmonic function in $(0, \infty))$ and $v(x)=u(x) / x$. Then the key transformation $T v$ in (12) can also be defined as the expectation over the $x$-process $\left\{X_{t}\right\}$ :

$$
T v(x)=c E^{x}\left\{\exp \int_{0}^{\infty} \frac{f\left(X_{t}, v\left(X_{t}\right) X_{t}\right)}{v\left(X_{t}\right) X_{t}} d t\right\},
$$

and the estimates (13) and (14) then follow from Khasmin'skii's lemma and Jensen's inequality, respectively.

\section{REFERENCES}

1. K.-S. Cheng and W.-M. Ni, On the structure of the conformal scalar curvature equation on $R^{n}$, Indiana Univ. Math. J. 41 (1992), 261-278.

2. E. Zeidler, Nonlinear functional analysis and its applications. I, Springer-Verlag, New York, 1986.

3. Z. Zhao, On the existence of positive solutions of nonlinear elliptic equations-a probabilistic potential theory approach, Duke Math. J. 69 (1993), 247-258.

Department of Mathematics, University of Missouri, Columbia, Missouri 65211

E-mail address: mathzz@mizzou1.edu 